\title{
Interactions of the Salience Network and Its Subsystems with the Default-Mode and the Central-Executive Networks in Normal Aging and Mild Cognitive Impairment
}

\author{
Ganesh B. Chand, Junjie Wu, Ihab Hajjar, ${ }^{1,3,{ }^{2}}$ and Deqiang Qiu${ }^{2,4, *}$
}

\begin{abstract}
Previous functional magnetic resonance imaging (fMRI) investigations suggest that the intrinsically organized largescale networks and the interaction between them might be crucial for cognitive activities. A triple network model, which consists of the default-mode network, salience network, and central-executive network, has been recently used to understand the connectivity patterns of the cognitively normal brains versus the brains with disorders. This model suggests that the salience network dynamically controls the default-mode and central-executive networks in healthy young individuals. However, the patterns of interactions have remained largely unknown in healthy aging or those with cognitive decline. In this study, we assess the patterns of interactions between the three networks using dynamical causal modeling in resting state fMRI data and compare them between subjects with normal cognition and mild cognitive impairment (MCI). In healthy elderly subjects, our analysis showed that the salience network, especially its dorsal subnetwork, modulates the interaction between the default-mode network and the centralexecutive network (Mann-Whitney $U$ test; $p<0.05$ ), which was consistent with the pattern of interaction reported in young adults. In contrast, this pattern of modulation by salience network was disrupted in MCI $(p<0.05)$. Furthermore, the degree of disruption in salience network control correlated significantly with lower overall cognitive performance measured by Montreal Cognitive Assessment $(r=0.295 ; p<0.05)$. This study suggests that a disruption of the salience network control, especially the dorsal salience network, over other networks provides a neuronal basis for cognitive decline and may be a candidate neuroimaging biomarker of cognitive impairment.
\end{abstract}

Keywords: dorsal and ventral salience networks; dynamical causal modeling; functional magnetic resonance imaging; large-scale network; mild cognitive impairment; normal aging

\section{Introduction}

$\mathbf{N}$ EUROIMAGING INVESTIGATIONS, including those using resting state functional magnetic resonance imaging (rsfMRI; Biswal et al., 1995; Raichle, 2015), in normal and diseased population have elucidated a set of large-scale, intrinsically organized brain networks (Deco et al., 2011; Power et al., 2011). Among them, the default-mode and centralexecutive networks have consistently been reported to have antagonistic activity in resting state or during task performing in healthy individuals (Chen et al., 2013; Fox et al., 2005; Fransson, 2005). Default-mode network is suggested to be more activated during internally directed cognitive activities, including self-monitoring and social functions, while the central-executive network is widely reported to be more activated for externally directed higher-order cognitive functions, such as attention, working memory, and decision-making (Bressler and Menon, 2010; Uddin, 2015). How the switch between these two networks is controlled dynamically is an active area of research.

A triple network model, comprising of these two antagonistic networks and the third network called the salience network (Chand and Dhamala, 2016b; Seeley et al., 2007; Uddin, 2015), has been recently proposed to understand the connectivity patterns of cognitively normal brain and the altered patterns in cognitive impairments (Chand et al., 2017;

\footnotetext{
${ }^{1}$ Division of Geriatrics and General Internal Medicine, Department of Medicine, Emory University School of Medicine, Atlanta, Georgia.

${ }^{2}$ Department of Radiology and Imaging Sciences, Emory University School of Medicine, Atlanta, Georgia.

${ }^{3}$ Department of Neurology, Emory Alzheimer's Disease Research Center, Emory University School of Medicine, Atlanta, Georgia.

${ }^{4}$ Department of Biomedical Engineering, Georgia Institute of Technology and Emory University, Atlanta, Georgia.

*These authors contributed equally.
} 
Menon, 2011). In cognitively normal young brains, the salience network was found to play a role in the dynamic switching of antagonistic activity between the defaultmode and central-executive networks (Chand and Dhamala, 2016a; Goulden et al., 2014; Sridharan et al., 2008). However, whether such dynamic switching retains in normal aging and/or alters in mild cognitive impairment (MCI) is unknown.

Recent studies further subdivide the salience network into the dorsal and ventral salience networks (Touroutoglou et al., 2012, 2016). Dorsal salience network is implicated to be important in attention and switching between cognitive resources, while the ventral system is suggested to be crucial during affections or emotions (Kurth et al., 2010; Touroutoglou et al., 2012, 2016). Whether the dorsal or ventral salience network subsystem controls over the default-mode and central-executive networks is largely unknown. Evaluating the network interactions in diseased or aging population could help test the hypothesis already mentioned and provide insights into the relationship between network interactions and cognitive functions. Despite a large body of literature evaluating single network activity that reported reduced default-mode network activity in MCI and Alzheimer's disease (Brier et al., 2014; Greicius and Kimmel, 2012; Greicius et al., 2004), the patterns of interactions between the triple networks have remained largely underinvestigated in normal healthy aging and MCI. Studying network interactions may provide insight into the neural mechanism in the progression of MCI to dementia or Alzheimer's disease (Hajjar et al., 2016; Iadecola, 2014; Oveisgharna and Hachinski, 2010).

Therefore, our objectives were to investigate the salience network control over the default-mode and central-executive networks in a group of patients with MCI using rsfMRI data in comparison to normal subjects and correlate the degree of disruption of this interaction with their global cognitive scores as measured by Montreal Cognitive Assessment (MoCA). As the salience network control is argued to be involved in maintaining task-related cognitive performance and/or resting state in cognitively healthy individuals (Menon, 2011; Sridharan et al., 2008), we hypothesized that this control will retain in normal cognitive aging, whereas the MCI group will demonstrate disruption in the control of salience network over the default-mode and central-executive networks and that the degree of disruption correlates with cognitive performance.

\section{Materials and Methods}

\section{Participants}

The study protocol was approved by the Institutional Review Board of Emory University, Atlanta, Georgia. The rsfMRI data from $54 \mathrm{MCI}$ individuals were obtained. The MCI participants inclusion criteria were as follows: (1) age $\geq 55$ years, (2) systolic blood pressure $\geq 140 \mathrm{~mm} \mathrm{Hg}$ or diastolic blood pressure $\geq 90 \mathrm{~mm} \mathrm{Hg}$ or receiving antihypertensive medications, and (3) executive MCI assessed by cognitive testing: MoCA $\leq 26$, cognitive performance at the 10th percentile or below in trail-mark or stroop interference or verbal fluency or abstractions, and minimal functional limitation as reflected by the functional assessment questionnaire $\leq 7$. The participants exclusion criteria were as follows: (1) systolic blood pressure $>200 \mathrm{~mm} \mathrm{Hg}$ or diastolic blood pressure $>110 \mathrm{~mm}$ $\mathrm{Hg}$, (2) renal disease or hyperkalemia, (3) active medical or psychiatric problems, (4) uncontrolled congestive heart failure (shortness of breath at rest or evidence of pulmonary edema on examination), (5) history of stroke in the past 3 years, (6) ineligibility for MRI (metal implants or cardiac pacemaker), (7) inability to complete cognitive test and MRI scan, (8) women of childbearing potential, and (9) diagnosis of dementia (selfreported or caregiver reported).

The MRI data from 20 normal participants were included based on the following criteria: age $\geq 50$ years, MoCA $\geq 26$, clinical dementia rating scale $(\mathrm{CDR})=0$, and normal logical memory subscale defined as $\geq 11$ for 16 or more years of education, $\geq 9$ for $8-15$ years of education, and $\geq 6$ for $<7$ years of education. The exclusion criteria were history of stroke in the past 3 years, ineligibility for MRI (metal implants or cardiac pacemaker), inability to complete cognitive test and MRI scan, and diagnosis of dementia of any type Abnormal thyroid-stimulating hormone $(>10)$ or B12 $(<250)$. All subjects were scanned on the same MRI scanner using the same imaging sequences. A written informed consent was collected from each participant before data collection.

\section{MRI acquisition}

MRI data were acquired on a SIEMENS Trio 3-Tesla scanner available at Center for Systems Imaging (CSI) of Emory University, Atlanta, Georgia. Foam padding and ear forms were used to limit head motion and reduce scanner noise to the subjects. High-resolution three-dimensional anatomical images were acquired using T1-weighted magnetizationprepared rapid gradient echo (MP-RAGE) sagittal with repetition time $(\mathrm{TR})=2300 \mathrm{~ms}$, echo time $(\mathrm{TE})=2.89 \mathrm{~ms}$, inversion time $=800 \mathrm{~ms}$, flip angle $(\mathrm{FA})=8$, resolution $=256 \times 256 \mathrm{ma}-$ trix, slices $=176$, thickness $=1 \mathrm{~mm}$. Blood oxygenation level dependent images for rsfMRI were collected axially using an echo-planar imaging sequence with $\mathrm{TR}=2500 \mathrm{~ms}, \mathrm{TE}=27 \mathrm{~ms}$, $\mathrm{FA}=90$, field of view $=22 \mathrm{~cm}$, resolution $=74 \times 74$ matrix, slices $=48$, thickness $=3 \mathrm{~mm}$, and bandwidth $=2598 \mathrm{~Hz} / \mathrm{Pixel}$. The subjects were instructed to hold still, keep their eyes open, and think nothing during the rsfMRI scan.

\section{Image preprocessing}

We preprocessed the MRI data using SPM12 (Wellcome Trust Centre for Neuroimaging, London, United Kingdom; www.fil.ion.ucl.ac.uk/spm/software/spm12). The preprocessing steps included slice-timing correction, motion correction, co-registration to individual anatomical image, normalization to the Montreal Neurological Institute (MNI) template, and finally spatial smoothing of the normalized images with a $6 \mathrm{~mm}$ isotropic Gaussian kernel. Out of $54 \mathrm{MCI}$ subjects, one subject was not included in the final analysis due to normalization failure. Among the remaining 53 MCI subjects, 51 subjects had motion $<3 \mathrm{~mm}$, and two subjects had motion $\sim 3.5 \mathrm{~mm}$.

\section{Independent component analysis}

We used constraint independent component analysis (ICA) to extract temporal signal for subsequence dynamical causal modeling (DCM) analysis. ICA has been widely used to investigate the functional brain network activities (Damoiseaux et al., 2012). A spatially constrained ICA approach has been introduced (Lin et al., 2010), which overcomes the difficulties in identifying components of interest and in determining the 
optimum number of components of conventional ICA. Constrained ICA has been highlighted as a useful tool, particularly if one is interested in specific brain areas or network(s). We used the Group ICA of fMRI Toolbox (GIFT; http:// mialab.mrn.org/software/gift) to compute ICA components.

We used the templates of each network (default-mode, salience, and central-executive networks) reported in a previous study (Shirer et al., 2012). For the dorsal and ventral salience networks, the coordinates from previous studies (Chand and Dhamala, 2016a; Sridharan et al., 2008; Touroutoglou et al., 2012) were used in the MarsBaR package (http://marsbar .sourceforge.net) to create the masks. We defined spherical regions with $6 \mathrm{~mm}$ radius based on MNI coordinates centered at the left dorsal anterior insula $(-36,21,1)$, right dorsal anterior insula $(36,21,1)$, and dorsal anterior cingulate cortex (4, $30,30)$ for the dorsal salience network. Similarly, for the ventral salience network we took spherical regions with $6 \mathrm{~mm}$ radius centered at the left ventral anterior insula $(-28,17$, $-15)$, right ventral anterior insula $(28,17,-15)$, and dorsal anterior cingulate cortex $(4,30,30)$. We then computed the network specific ICA components. This approach has been previously suggested to render better representations of networks compared to choosing the average or first eigenvariate of a template (Craddock et al., 2012; Goulden et al., 2014; Shirer et al., 2012; Smith et al., 2011). We then used the time courses (components) produced by the constrained ICA for subsequent DCM analysis.

\section{Dynamical causal modeling}

We used DCM to evaluate the dynamic interaction between the three networks. DCM analysis (Friston et al., 2003) assesses the directed connectivity between different brain areas or networks. In DCM analysis, several hypothesized possible models are specified, and Bayesian model selection (BMS) is then used to infer the model that best fits the measured data (Stephan et al., 2010). Recent methodological advances in DCM facilitate the implementation of random effects (RFX) for model selection (Stephan et al., 2009), which allows the specification of nonlinear modulations to investigate how a brain area (or network) influences connection strengths between other brain areas (or networks; $\mathrm{Li}$ et al., 2011; Stephan et al., 2008) and make use of stochastic models (Daunizeau et al., 2012). The stochastic model accounts for noise more accurately and, therefore, allows the application of DCM to rsfMRI data (Daunizeau et al., 2012; Friston et al., 2014). We carried out DCM analysis using SPM12 (Wellcome Trust Centre for Neuroimaging).

We specified three models for each subject with fully connected intrinsic connections (Friston et al., 2003, 2014) between the networks (Fig. 3). Model 1 specified nonlinear modulation by the default-mode network on both reciprocal connections between the salience and central-executive networks. Model 2 specified nonlinear modulation by the salience network on both connections between the defaultmode and central-executive networks. Finally, model 3 specified nonlinear modulation by the central executive on both connections between the default-mode and salience networks. In this study, we used nonlinear differential equation based stochastic DCM already described to estimate the models. For both MCI and control groups, we identified the model with the highest probability using both the fixed effects
(FFX) and the RFX-BMS methods, respectively. Both methods provide complementary information, but differ on the underlying assumptions. The FFX-BMS assumes that the optimal model is homogeneous across subjects and uses the group log-evidence, which measures the balance between fit and complexity of models and quantifies the relative goodness of models. In contrast, the RFX-BMS accounts for heterogeneity of model structure across subjects. The RFXBMS provides posterior model probability, which measures how likely a specific model generated the data of randomly selected subject, and exceedance probability, which measures how one model is more likely than any other model, in the group data (Stephan et al., 2010).

\section{Statistical analysis}

Baseline factors were compared between the normal control (NC) and MCI groups using Mann-Whitney $U$ test. Pearson's correlation analysis was performed to assess the association between connectivity strength of network modulations with MoCA for each model in MCI subjects only. We limited this analysis to MCI because all subjects in the control subjects had normal MoCA. All data were analyzed using MATLAB (Natick, MA; https://www.mathworks.com).

\section{Results}

Fifty-three MCI and 20 normal subjects were included in the analysis. Sample characteristics are provided in Table 1. In the MCI group, mean age was 66.9 years (standard deviation [SD] 8.1), $60 \%$ were African American, $52.8 \%$ were women, mean education was 15 years (SD 2.4), mean systolic blood pressure $150.7 \mathrm{~mm}$ of $\mathrm{Hg}$ (SD 21.3), and mean diastolic blood pressure $90.9 \mathrm{~mm}$ of $\mathrm{Hg}$ (SD 13.5). In normal group, mean age was 65.8 years (SD 8.8), 20\% were African American, $70 \%$ were women, and eight had hypertension. The mean education was 16 years (SD 2.9), mean systolic blood pressure $128.8 \mathrm{~mm}$ of $\mathrm{Hg}$ (SD 23.1), and mean diastolic blood pressure $71.7 \mathrm{~mm}$ of $\mathrm{Hg}$ (SD 11.7). Figure 1 shows

Table 1. Characteristics of the Mild Cognitive Impairment and Normal Control Samples

\begin{tabular}{lccc}
\hline Characteristic & $\begin{array}{c}\text { MCI total } \\
\text { sample }\end{array}$ & $\begin{array}{c}\text { NC total } \\
\text { sample }\end{array}$ & $\mathrm{p}$ \\
\hline $\mathrm{n}$ & 53 & 20 & \\
Age, years & $66.9(8.1)$ & $65.8(8.8)$ & 0.776 \\
$\begin{array}{l}\text { Sex, women } \\
\text { Race }\end{array}$ & $28(52.8 \%)$ & $14(70 \%)$ & 0.186 \\
$\quad$ Black & $32(60 \%)$ & $4(20 \%)$ & \\
$\quad$ White & $19(36 \%)$ & $16(80 \%)$ & \\
$\quad$ Other & $2(4 \%)$ & & \\
$\begin{array}{l}\text { Education, } \\
\quad \text { years }\end{array}$ & $15(2.4)$ & $16(2.9)$ & 0.284 \\
$\begin{array}{c}\text { Systolic BP, } \\
\quad \text { mm Hg }\end{array}$ & $150.7(21.3)$ & $128.8(23.1)$ & $<10^{-3}$ \\
$\begin{array}{c}\text { Diastolic BP, } \\
\quad \text { mm Hg }\end{array}$ & $90.9(13.5)$ & $71.7(11.7)$ & $<10^{-6}$ \\
\hline
\end{tabular}

Number represents mean (SD) or count (\%); $p$-value represents MCI versus NC using Mann-Whitney $U$ test or chi-square test; and $p<0.05$ is considered statistically significant.

$\mathrm{BP}$, blood pressure; MCI, mild cognitive impairment; NC, normal control; SD, standard deviation. 
A DMN

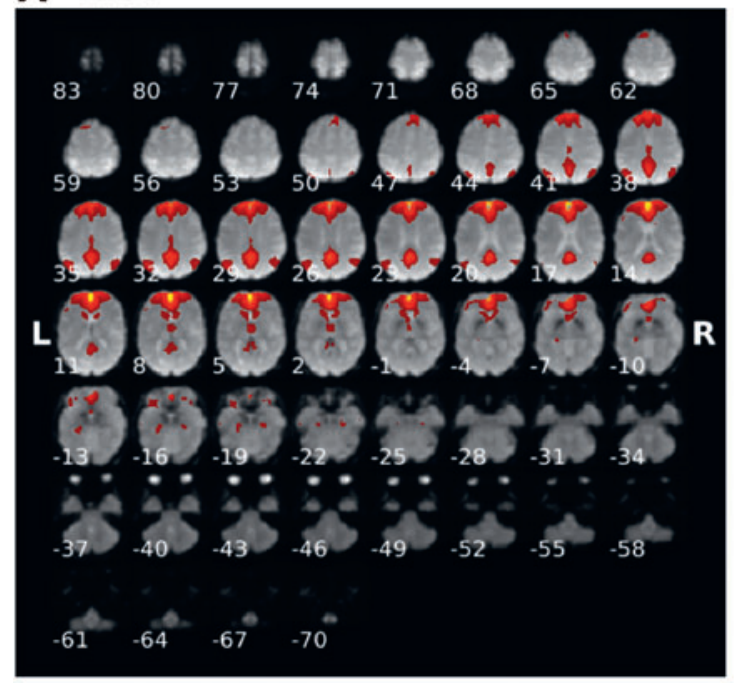

\section{CEN}

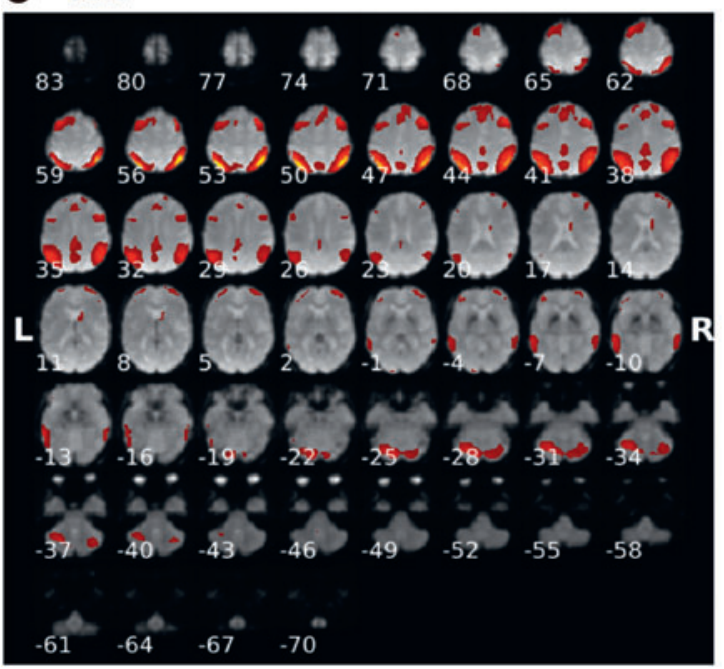

\section{B SN}
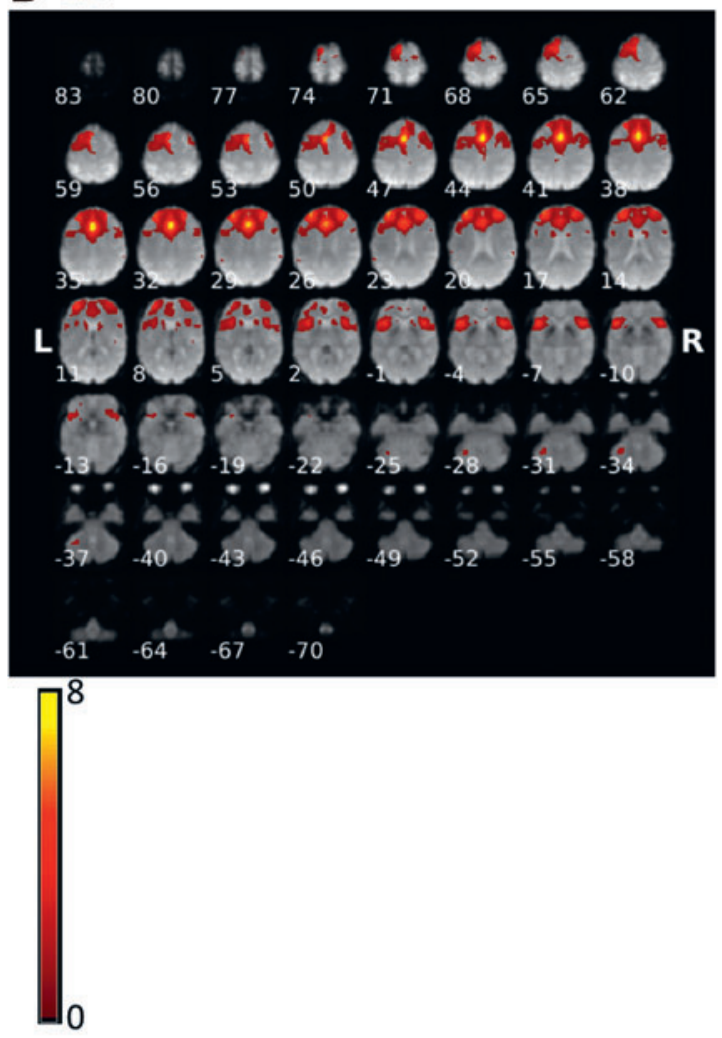

FIG. 1. The $t$-value maps of (A) the DMN, (B) the SN, and (C) the CEN from the constrained ICA overlaid on mean BOLD images in MCI. BOLD, blood oxygenation level dependent; CEN, central-executive network; DMN, default-mode network; ICA, independent component analysis; MCI, mild cognitive impairment; SN, salience network. Color images available online at www.liebertpub.com/brain

the results of the constrained ICA for the (Fig. 1A) defaultmode network, (Fig. 1B) salience network, and (Fig. 1C) central-executive network components for MCI. Figure 2 displays the results of constrained ICA for normal group.

Three network connection models were considered and compared statistically and are illustrated in Figure 3. For model 1 (Fig. 3A), the default-mode network extends modulations over the connections between the salience network and the central-executive network; for model 2 (Fig. 3B), the salience network extends modulations over the connections between the default-mode network and the centralexecutive network suggesting controlling/switching role of the salience network; for model 3 (Fig. 3C), the centralexecutive network extends modulations over the connection between the default-mode network and the salience network.

We carried out the RFX-BMS analysis (Fig. 4). The first column (Fig. 4A, B) shows the expected posterior model probability and exceedance probability for normal group Similarly, the second column (Fig. 4C, D) shows the expected posterior probability and exceedance probability for MCI group. The RFX analysis revealed that model 2 has higher probability value than other models in normal group. It also revealed that model 2 no longer has higher probability in MCI group, instead the overall probability values shifted toward model 3 . The three models were further compared within the normal control, MCI, and between the normal control and MCI groups, respectively (Fig. 5). In normal control (Fig. 5A), we found that model 2 had significantly higher probability than model 1 ( $p=0.046$; Mann-Whitney $U$ test) and model $3(p=0.002)$, but there was no significant difference between models 1 and 3 ( $p=0.531$ ). In MCI group, we found that model 3 had significantly higher probability than model $1\left(p=8.53 \times 10^{-19}\right)$ and model $2(p=7.81 \times$ $\left.10^{-18}\right)$, and model 2 has higher probability than model 1 
A DMN

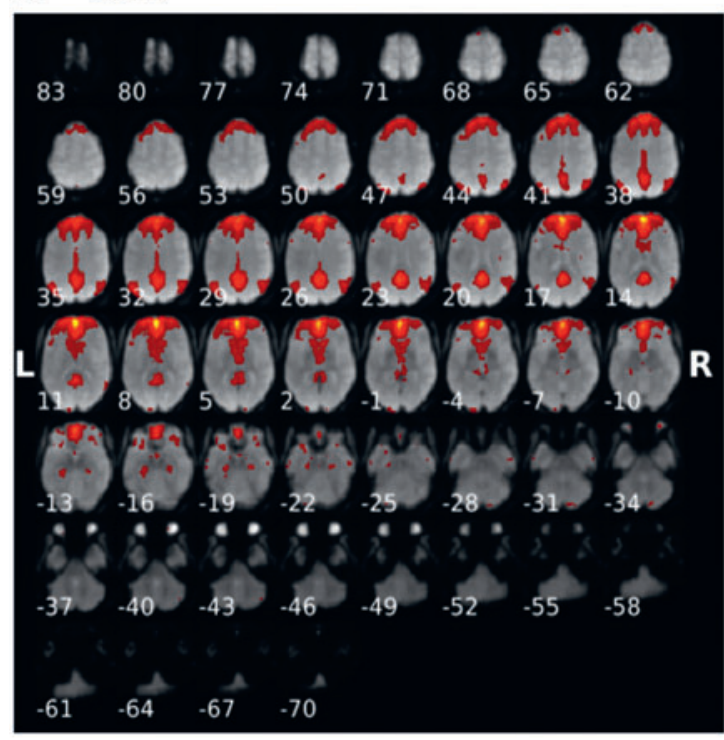

C CEN

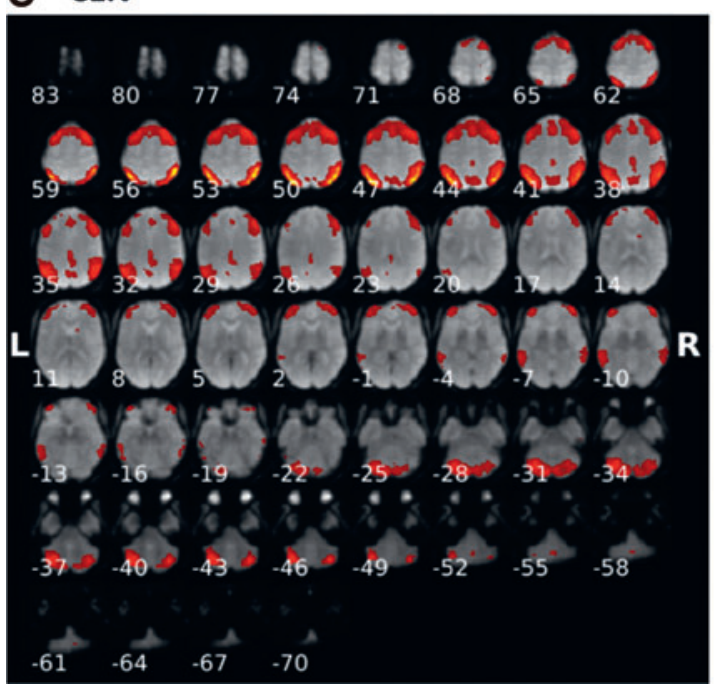

B SN

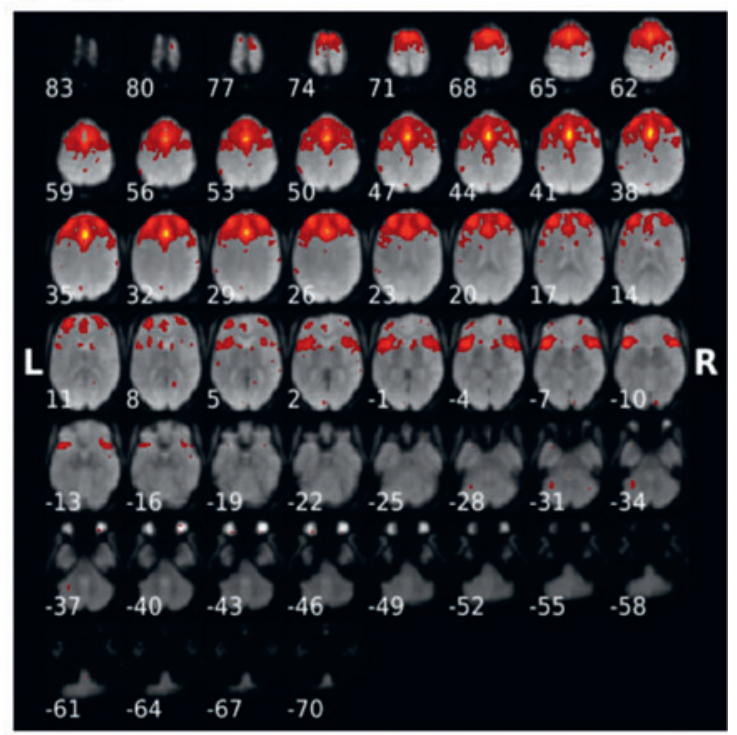

7.5

0

FIG. 2. The $t$-value maps of (A) the DMN, (B) the $\mathrm{SN}$, and (C) the CEN from the constrained ICA overlaid on mean BOLD images in NC. NC, normal control. Color images available online at www.liebertpub.com/brain

$\left(p=1.91 \times 10^{-4}\right)$. Comparison between normal and MCI groups demonstrated a significant difference between two groups in each model (Table 2).

To validate our findings, the FFX analysis was also performed and we found similar patterns of network interactions that model 2 had higher probability in normal controls and model 3 in MCI group (Supplementary Fig. S1; Supplementary Data are available online at www.liebertpub.com/brain). To evaluate whether the observation was related to hypertension status or cognitive impairment itself, we further compared the network models between two groups of cognitively normal subject, one hypertensive $(n=8)$ and the other normotensive $(n=12)$. No significant difference related to hypertension status was found (Supplementary Fig. S2).

We further investigated the interactions of the salience network subsystems - the dorsal salience network and ventral salience network-with the default-mode and central- executive networks. We observed that the dorsal salience network exhibits control over the default-mode and centralexecutive networks in the normal group, but not in the MCI group (Fig. 6 for RFX results; Supplementary Fig. S3 for FFX results). In contrast, we also found that the ventral salience network does not exhibit control over the defaultmode and central-executive networks in neither the normal group nor the MCI group (Fig. 7 for RFX results; Supplementary Fig. S4 for FFX results).

\section{rsfMRI-cognition relation}

We investigated the association between connectivity strength and MoCA score in patients with MCI (since normal group all had normal scores). Lower modulation strength from salience network on the other two networks derived from DCM analysis was associated with worse performance on 


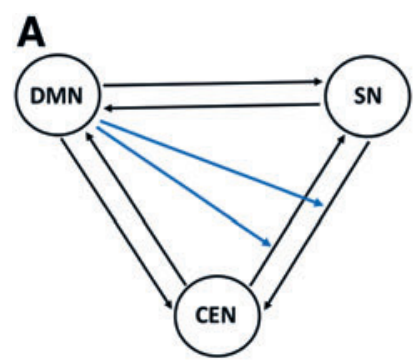

Model 1: DMN Modulations

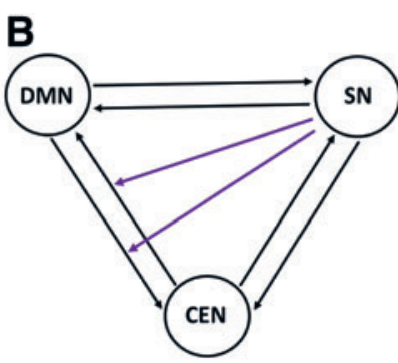

Model 2: SN Modulations

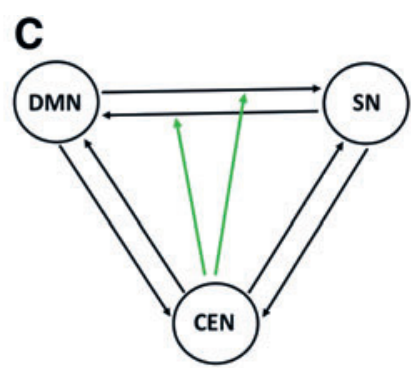

Model 3: CEN Modulations

FIG. 3. Model representation: (A) Model 1 specifies nonlinear modulation by the DMN on both connections between the SN and CEN, (B) model 2 specifies nonlinear modulation by the SN on both connections between the DMN and CEN, and (C) model 3 specifies nonlinear modulation by the CEN on both connections between the DMN and SN. Color images available online at www.liebertpub.com/brain

cognitive testing as reflected by the MoCA scores ( $p=$ $0.034 ; r=0.295)$. These results are presented in Figure 8.

\section{Discussion}

The present study showed that MCI is associated with a disrupted interaction among the default-mode, salience (dorsal salience), and central-executive networks, whereas it is maintained in cognitively normal elderly subjects. Interactions be- tween the networks have been argued to be important for effective maintenance of normal cognitive status (Bressler and Menon, 2010; Chand and Dhamala, 2016a; Menon, 2015; Uddin, 2015). It is consistently reported that the salience network drives the default-mode and central-executive networks during both resting state and tasks in healthy younger population (Chand and Dhamala, 2016a; Goulden et al., 2014; Sridharan et al., 2008). Our results for healthy older (normal) group are consistent with those findings, suggesting that the
FIG. 4. The first column (A, B) displays the RFX results in terms of expected and exceedance probabilities in $\mathrm{NC}$ group indicating that model 2 has a higher probability than other models. The second column $(\mathbf{C}, \mathbf{D})$ displays the fixed effect results in MCI group demonstrating that model 2 no longer has a higher probability, instead it shifted to model 3. RFX, random effects.
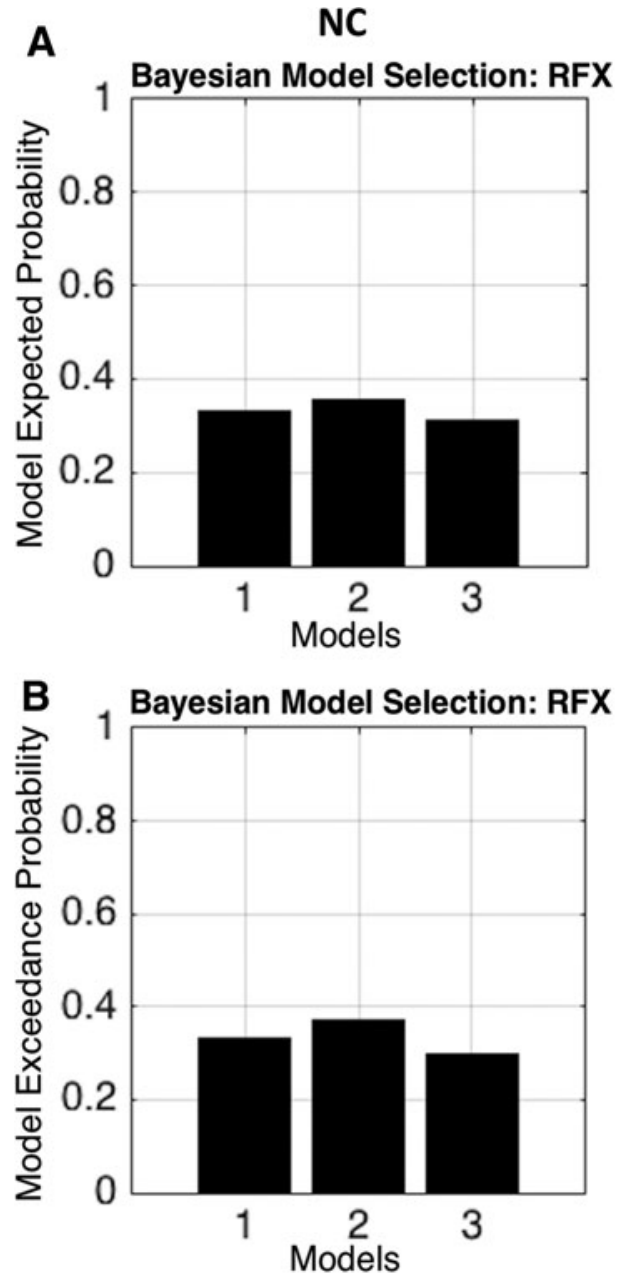
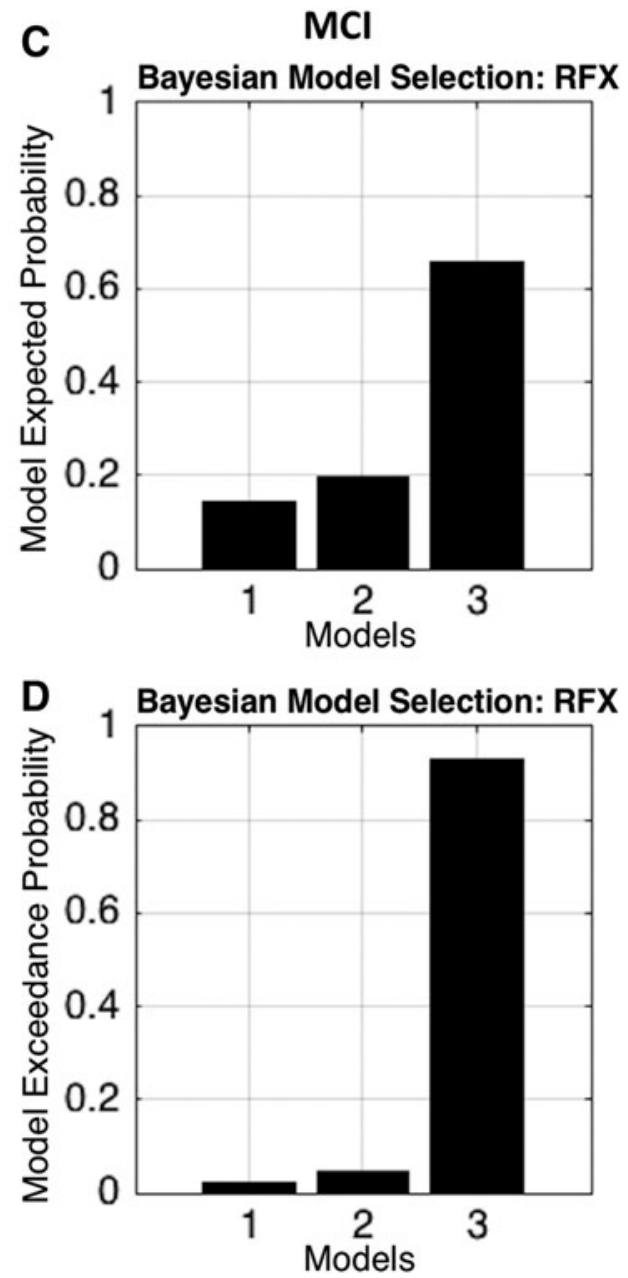

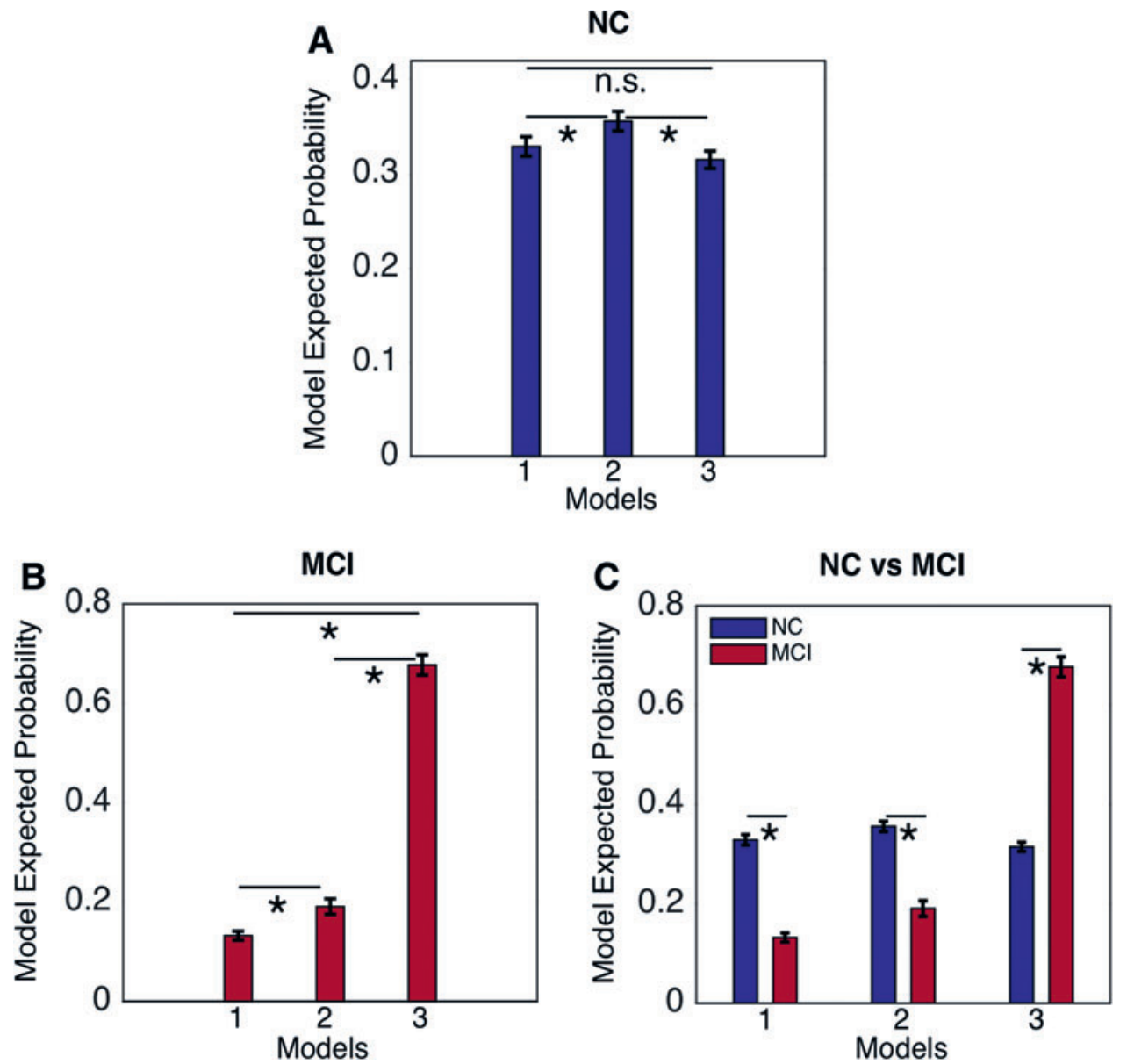

FIG. 5. Comparison of models within groups and between groups: (A) comparison between the models within NC group shows significantly higher probability of model 2, (B) comparison between the models within MCI group shows that model 2 no longer has higher probability, instead model 3 has, and $(\mathbf{C})$ comparison between $\mathrm{NC}$ and $\mathrm{MCI}$ in each model. *Indicates $p<0.05$; FDRcorrected. n.s., not significant; FDR, false discovery rate. Color images available online at www.liebertpub .com/brain dynamic switching role of salience network is intact in healthy aging. In addition, a comparison between hypertensive and normotensive cognitively normal individuals showed that there is no significant difference in the DCM model probability (Supplementary Fig. S1). We also found significant correlation between the modulation strength of the salience network over the other two networks and the neuropsychological test. These findings suggest that the difference in network interaction found in our MCI group is likely primarily driven by neuronal changes associated with cognitive impairment rather than vascular contributions due to hypertension in the MCI group.

Previous research has established that the default-mode network activity is impaired in MCI and Alzheimer's disease (Brier et al., 2014; Greicius and Kimmel, 2012; Greicius et al., 2004). Our results for MCI further demonstrated impaired control of the salience network over the default-

Table 2. Model Comparison Between THE Normal Control and THE Mild COGNITIVE IMPAIRMENT GROUPS

\begin{tabular}{lccc}
\hline & $\begin{array}{c}\text { NC mean } \\
(S D)\end{array}$ & $\begin{array}{c}\text { MCI mean } \\
(S D)\end{array}$ & $\begin{array}{c}\text { NC vs. } \\
\text { MCI } \mathrm{p} \text {-value }\end{array}$ \\
\hline Model 1 & $0.329(0.045)$ & $0.135(0.065)$ & $8.61 \times 10^{-11}$ \\
Model 2 & $0.356(0.045)$ & $0.191(0.114)$ & $8.26 \times 10^{-09}$ \\
Model 3 & $0.315(0.040)$ & $0.677(0.146)$ & $4.08 \times 10^{-10}$ \\
\hline
\end{tabular}

mode and central-executive networks in this population, which may underlie reduced default-mode network activity. Our study was inspired by several studies that implemented network analysis in aging research. Recent study by Tsvetanov et al. (2016) reports the directed connectivity within and between the salience, dorsal attention, and defaultmode networks. Our study consisted of central-executive network instead of dorsal attention network and tested a different hypothesis that whether the salience network is responsible in switching between the default-mode and central-executive networks, which was tested for healthy younger adults in previous studies (Goulden et al., 2014; Sridharan et al., 2008). In addition, we included the nonlinear modulations in interactions as implemented in prior investigations (Goulden et al., 2014; Li et al., 2011; Stephan et al., 2008).

It is interesting to note that the salience network-based control feature might be facilitated by unique cytoarchitecture of its key regions, the anterior insula and the dorsal anterior cingulate cortex (Bonnelle et al., 2012). These regions consist of von Economo neurons. These specific types of neurons are present in larger number in humans than in great apes, but not at all in other primates, and are suggested to relay information processed in these regions to the other parts of the brain (Allman et al., 2005, 2010; Watson et al., 2006). Whether these neurons are specific to the switching function of the salience network remains to be established. Previous studies have consistently reported co-activation of 
FIG. 6. (A) Model 1 specifies nonlinear modulation by the DMN on both connections between the dSN and CEN, (B) model 2 specifies nonlinear modulation by the dSN on both connections between the DMN and CEN, and (C) model 3 specifies nonlinear modulation by the CEN on both connections between the DMN and dSN. The panels $(\mathbf{D}, \mathbf{E})$ display the RFX results in terms of expected and exceedance probabilities in NC group indicating that model 2 has a higher probability than other models. The panels $(\mathbf{F}, \mathbf{G})$ display the RFX results in MCI group demonstrating that model 2 no longer has a higher probability; instead it shifted to model 3. dSN, dorsal salience network. Color images available online at www.liebertpub.com/brain
A

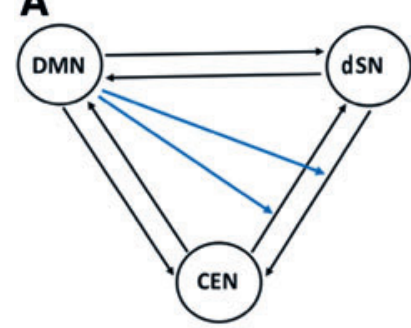

Model 1: DMN modulations

D

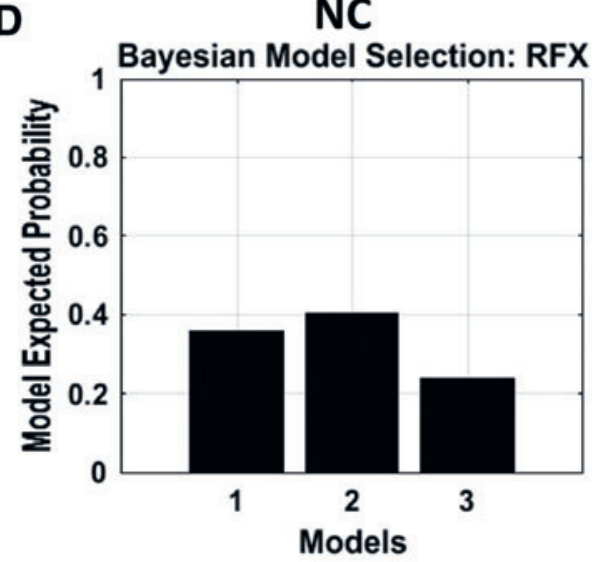

E

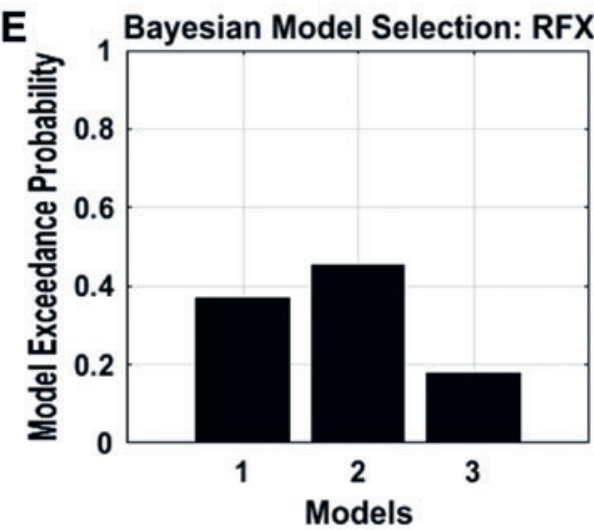

B

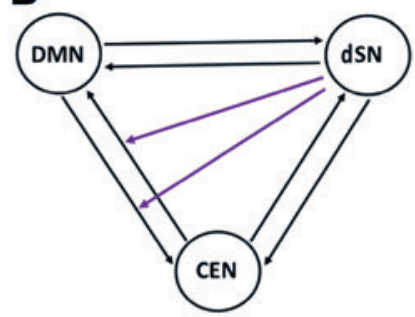

Model 2: d SN modulations

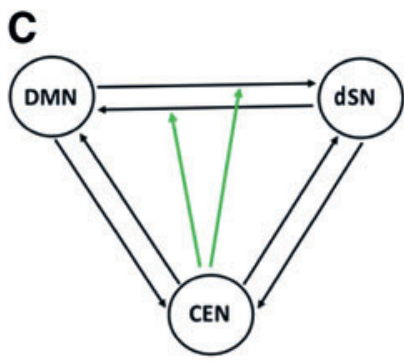

Model 3: CEN modulations

$\mathrm{MCl}$
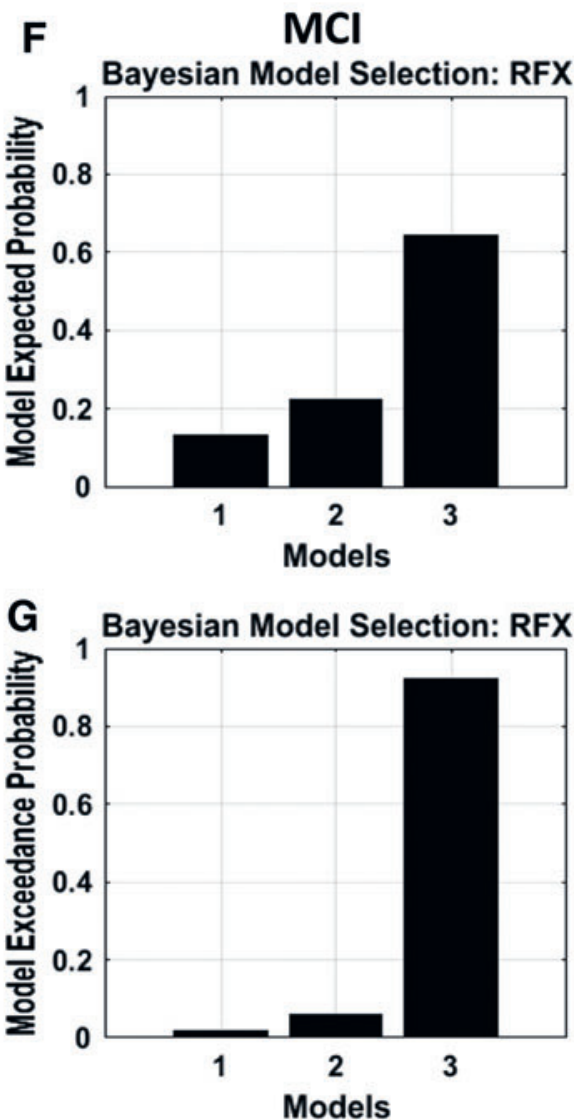

these regions (Chand and Dhamala, 2016b, 2017; Dosenbach et al., 2006; Seeley et al., 2007). The anterior insula is functionally connected to the networks responsible for adaptive behavior, including the salience network (Seeley et al., 2007) and the frontoparietal control network (Vincent et al., 2008). This area has direct white matter connections to other key regions, including the dorsal anterior cingulate cortex (Jilka et al., 2014), the inferior parietal lobe (Uddin et al., 2010), and the temporoparietal junction (Kucyi et al., 2012), making the insula well placed to perform its putative role of evaluating the environment and the cognitive state (Uddin et al., 2010), reorienting of attention (Ullsperger et al., 2010), and switching between cognitive resources (Uddin and Menon, 2009).

The dorsal anterior insula-a key node of the dorsal salience network - is reciprocally connected with multiple regions in prefrontal cortex, precentral operculum, parietal, and temporal cortices (Cerliani et al., 2012; Mesulam and
Mufson, 1982a, b; Mufson and Mesulam, 1982) and is implicated to be important in attention and switching between cognitive resources (Sridharan et al., 2008; Touroutoglou et al., 2012). In contrast, the ventral anterior insula-a key node of the ventral salience network-is reciprocally connected with multiple limbic and paralimbic structures (Cerliani et al., 2012; Mesulam and Mufson, 1982a, b; Mufson and Mesulam, 1982) and is suggested to be crucial during affections or emotions (Kurth et al., 2010; Touroutoglou et al., 2012, 2016). The activity in the dorsal anterior cingulate cortex is known to signal the need for enhanced cognitive control and to implement behavioral changes (Egner, 2009). Our results for normal elderly control therefore provide strong evidence for the neural basis of the control feature of the salience network (Allman et al., 2005, 2010; Watson et al., 2006), consistent with previous reports in normal young subjects (Chand and Dhamala, 2016a; Goulden et al., 2014; Sridharan et al., 2008). Emerging evidence 


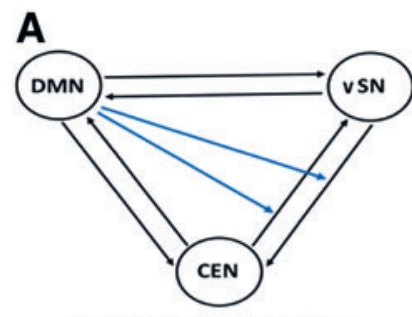

Model 1: DMN modulations

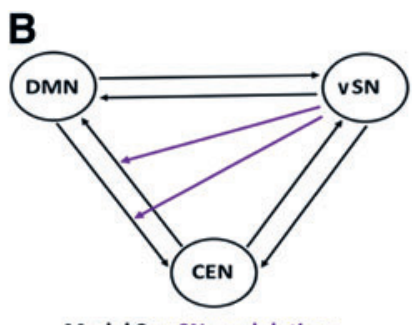

Model 2: v SN modulations

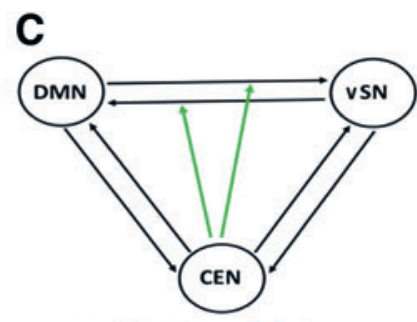

Model 3: CEN modulations
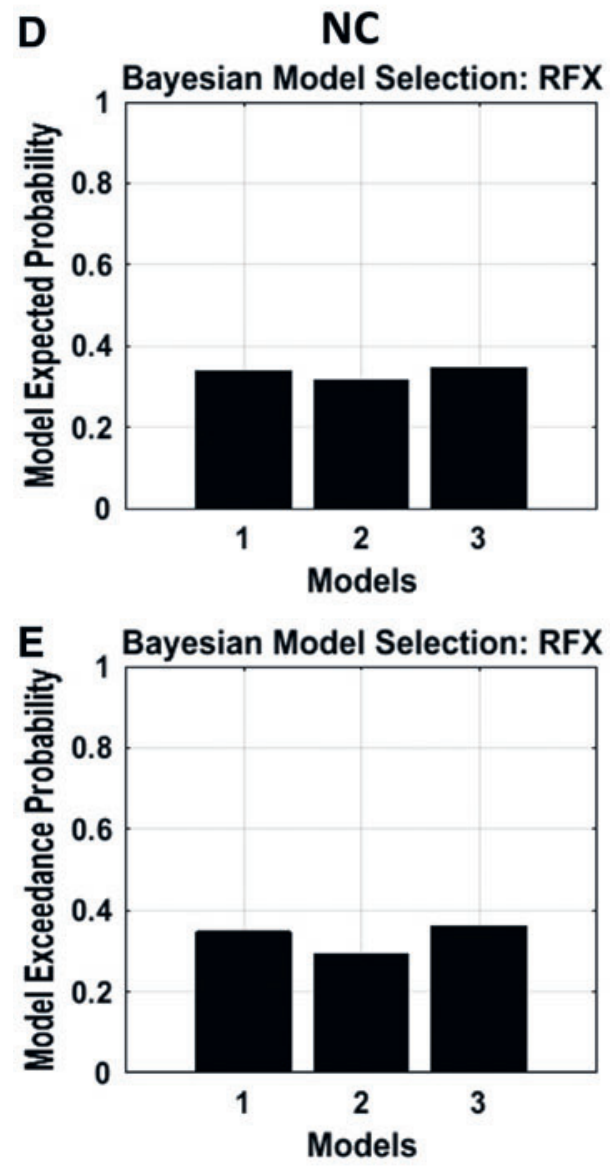
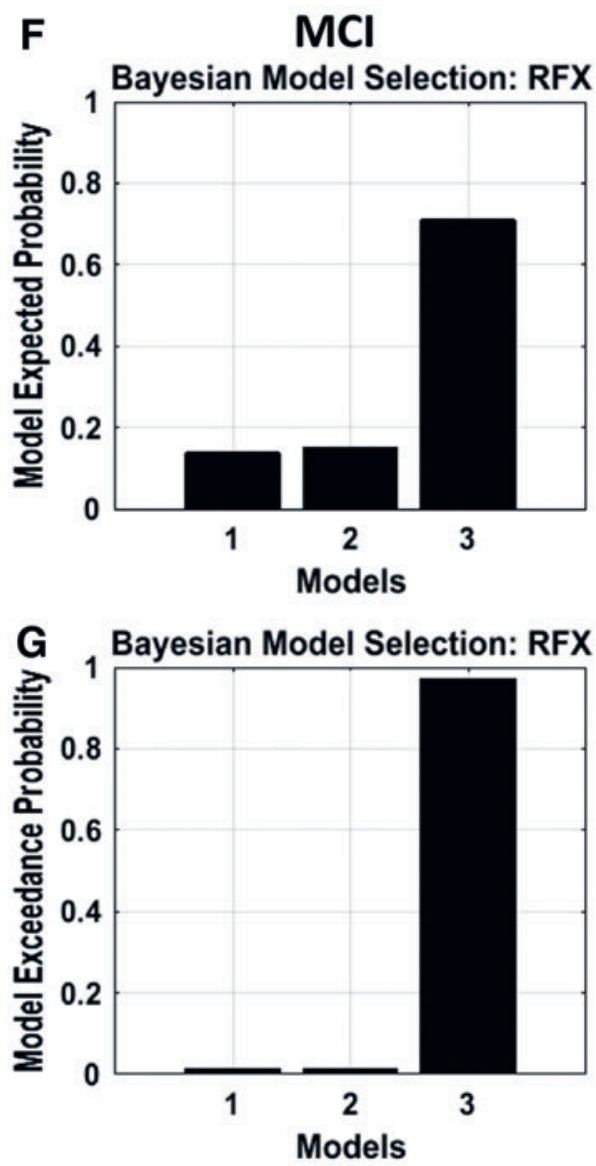

FIG. 7. (A) Model 1 specifies nonlinear modulation by the DMN on both connections between the vSN and CEN, (B) model 2 specifies nonlinear modulation by the vSN on both connections between the DMN and CEN, and (C) model 3 specifies nonlinear modulation by the CEN on both connections between the DMN and vSN. The panels (D, E) display the RFX results in terms of expected and exceedance probabilities in NC group indicating that model 2 no longer has a higher probability. The panels $(\mathbf{F}, \mathbf{G})$ display the RFX results in MCI group demonstrating that model 2 no longer has a higher probability; instead it shifted to model 3. vSN, ventral salience network. Color images available online at www.liebertpub.com/brain shows atypical engagement of the salience network in several neuropsychiatric disorders, such as in autism spectrum disorder, schizophrenia, and frontotemporal dementia (Menon, 2015; Uddin, 2015).

Prior studies report that the default-mode network activity decreases in MCI and/or with progression of Alzheimer's disease (Brier et al., 2014; Greicius and Kimmel, 2012; Greicius et al., 2004). Decreased probability of model 1 (i.e., modulations by default-mode network) in MCI compared to that of $\mathrm{NC}$ might indicate such changes as suggested by those studies. Dorsolateral prefrontal cortex - a key node of the central-executive network-has rich connections with several areas in the brain such as visual, somatosensory, and auditory areas and receives the information from occipital, parietal, and temporal cortices and is shown to be important in several cognitive functions, including executive function (Chand and Dhamala, 2017; Chand et al., 2016; Miller and Cohen, 2001; Petrides and Pandya, 1999).
The central-executive network activity has been conflicted with disease (Diener et al., 2012). These works suggest this network, especially the dorsolateral prefrontal cortex node, to hypoactivate or hyperactivate with disease. Our results in MCI suggested that the probability of model 3 (i.e., modulations by central-executive network) is greater in MCI compared to that of NC implying hypermodulations by central-executive network. Our findings and existing literature taken together thus suggest that the salience network's control mechanism over default-mode and centralexecutive networks is achieved in healthy aging; however, this control is impaired and the network modulations are altered in MCI.

In conclusion, this study evaluated the resting-state patterns of directed interactions among the default-mode, salience, and central-executive networks in aging population with normal cognition and those with MCI. We found that the salience network, especially the dorsal salience network, 
FIG. 8. The correlation analysis between the network connections and MoCA scores in MCI group. The first row shows the correlation of connectivity of DMN on the connections between $\mathrm{SN}$ and CEN with MoCA, the second row shows the correlation of connectivity of SN on the connections between DMN and CEN with MoCA, and the third row shows the correlation of connectivity of CEN on the connections between DMN and SN with MoCA. MoCA, Montreal Cognitive Assessment. Color images available online at www .liebertpub.com/brain
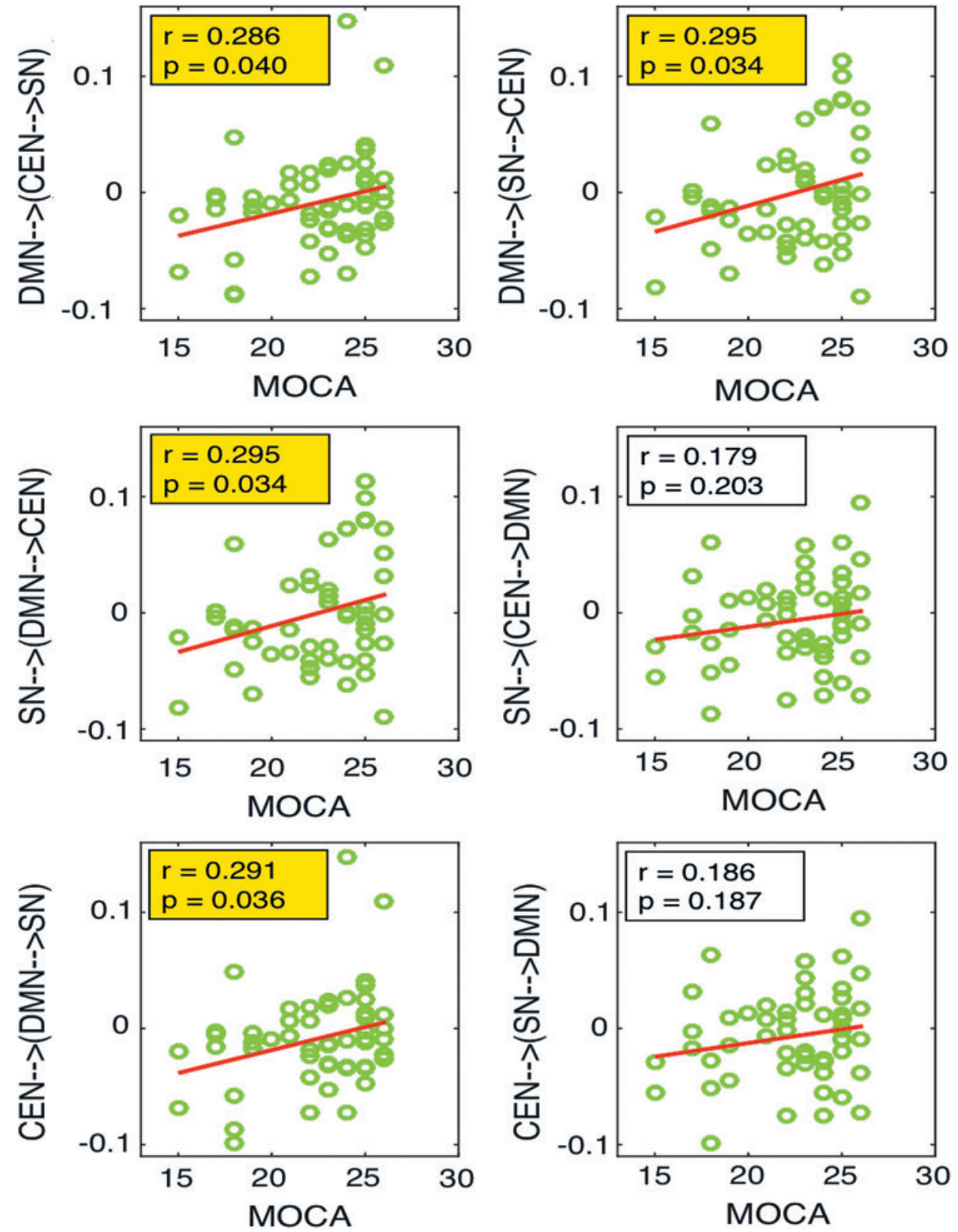

played causal control over the default-mode and centralexecutive networks for normal control and that this feature was disrupted in MCI. These findings advance our current understanding of how the default-mode, salience, and centralexecutive networks coordinate neural information in healthy aging and in MCI.

\section{Acknowledgments}

The authors thank the members of our team and the subjects who volunteered for these studies. This research was conducted with NIA/NIH grants RF1AG051633 and R01AG042127 to I.H. D.Q. is supported by NIH grants AG25688, AG42127, AG49752, and AG51633 and has received research support from Medtronic and Siemens Medical Solutions.

\section{Author Disclosure Statement}

All authors have approved the article and agree with submission to Brain Connectivity. We have read and have abided by the statement of ethical standards for manuscripts submitted to Brain Connectivity.

\section{References}

Allman JM, Tetreault NA, Hakeem AY, Manaye KF, Semendeferi K, Erwin JM, et al. 2010. The von Economo neurons in frontoinsular and anterior cingulate cortex in great apes and humans. Brain Struct Funct 214:495-517.

Allman JM, Watson KK, Tetreault NA, Hakeem AY. 2005. Intuition and autism: a possible role for von Economo neurons. Trends Cogn Sci 9:367-373. 
Biswal B, Haughton VM, Hyde J. 1995. Functional connectivity in the motor cortex resting human brain using echo-planar MRI. Magn Reson Med 34:537-541.

Bonnelle V, Ham TE, Leech R, Kinnunen KM, Mehta MA, Greenwood RJ, Sharp DJ. 2012. Salience network integrity predicts default mode network function after traumatic brain injury. Proc Natl Acad Sci U S A 109:4690-4695.

Bressler SL, Menon V. 2010. Large-scale brain networks in cognition: emerging methods and principles. Trends Cogn Sci 14: 277-290.

Brier MR, Thomas JB, Ances BM. 2014. Network dysfunction in Alzheimer's disease: refining the disconnection hypothesis. Brain Connect 4:299-311.

Cerliani L, Thomas RM, Jbabdi S, Siero JC, Nanetti L, Crippa A, et al. 2012. Probabilistic tractography recovers a rostrocaudal trajectory of connectivity variability in the human insular cortex. Hum Brain Mapp 33:2005-2034.

Chand GB, Dhamala M. 2016a. Interactions among the brain default-mode, salience, and central-executive networks during perceptual decision-making of moving dots. Brain Connect 6:249-254.

Chand GB, Dhamala M. 2016b. The salience network dynamics in perceptual decision-making. Neuroimage 134:85-93.

Chand GB, Dhamala M. 2017. Interactions between the anterior cingulate-insula network and the fronto-parietal network during perceptual decision-making. Neuroimage 152: 381-389.

Chand GB, Lamichhane B, Dhamala M. 2016. Face or house image perception: beta and gamma bands of oscillations in brain networks carry out decision-making. Brain Connect 6: $621-631$.

Chand GB, Wu J, Qiu D, Hajjar I. 2017. Racial differences in insular connectivity and thickness and related cognitive impairment in hypertension. Front Aging Neurosci 9:177.

Chen AC, Oathes DJ, Chang C, Bradley T, Zhou ZW, Williams LM, et al. 2013. Causal interactions between fronto-parietal central executive and default-mode networks in humans. Proc Natl Acad Sci U S A 110:19944-19949.

Craddock RC, James GA, Holtzheimer PE, 3rd, Hu XP, Mayberg HS. 2012. A whole brain fMRI atlas generated via spatially constrained spectral clustering. Hum Brain Mapp 33: 1914-1928.

Damoiseaux JS, Prater KE, Miller BL, Greicius MD. 2012. Functional connectivity tracks clinical deterioration in $\mathrm{Alz}$ heimer's disease. Neurobiol Aging 33:828 e819-e830.

Daunizeau J, Stephan KE, Friston KJ. 2012. Stochastic dynamic causal modelling of fMRI data: should we care about neural noise? Neuroimage 62:464-481.

Deco G, Jirsa VK, McIntosh AR. 2011. Emerging concepts for the dynamical organization of resting-state activity in the brain. Nat Rev Neurosci 12:43-56.

Diener C, Kuehner C, Brusniak W, Ubl B, Wessa M, Flor H. 2012. A meta-analysis of neurofunctional imaging studies of emotion and cognition in major depression. Neuroimage 61:677-685.

Dosenbach NU, Visscher KM, Palmer ED, Miezin FM, Wenger $\mathrm{KK}$, Kang HC, et al. 2006. A core system for the implementation of task sets. Neuron 50:799-812.

Egner T. 2009. Prefrontal cortex and cognitive control: motivating functional hierarchies. Nat Neurosci 12:821-822.

Fox MD, Snyder AZ, Vincent JL, Corbetta M, Van Essen DC, Raichle ME. 2005. The human brain is intrinsically organized into dynamic, anticorrelated functional networks. Proc Natl Acad Sci U S A 102:9673-9678.
Fransson P. 2005. Spontaneous low-frequency BOLD signal fluctuations: an fMRI investigation of the resting-state default mode of brain function hypothesis. Hum Brain Mapp 26:15-29.

Friston KJ, Harrison L, Penny W. 2003. Dynamic causal modelling. Neuroimage 19:1273-1302.

Friston KJ, Kahan J, Biswal B, Razi A. 2014. A DCM for resting state fMRI. Neuroimage 94:396-407.

Goulden N, Khusnulina A, Davis NJ, Bracewell RM, Bokde AL, McNulty JP, Mullins PG. 2014. The salience network is responsible for switching between the default mode network and the central executive network: replication from DCM. Neuroimage 99:180-190.

Greicius MD, Kimmel DL. 2012. Neuroimaging insights into network-based neurodegeneration. Curr Opin Neurol 25: 727-734

Greicius MD, Srivastava G, Reiss AL, Menon V. 2004. Defaultmode network activity distinguishes Alzheimer's disease from healthy aging: evidence from functional MRI. Proc Natl Acad Sci U S A 101:4637-4642.

Hajjar I, Goldstein FC, Martin GS, Quyyumi AA. 2016. Roles of arterial stiffness and blood pressure in hypertensionassociated cognitive decline in healthy adults. Hypertension 67:171-175

Iadecola C. 2014. Hypertension and dementia. Hypertension 64: $3-5$.

Jilka SR, Scott G, Ham T, Pickering A, Bonnelle V, Braga RM, et al. 2014. Damage to the salience network and interactions with the default mode network. J Neurosci 34:10798-10807.

Kucyi A, Moayedi M, Weissman-Fogel I, Hodaie M, Davis KD. 2012. Hemispheric asymmetry in white matter connectivity of the temporoparietal junction with the insula and prefrontal cortex. PLoS One 7:e35589.

Kurth F, Zilles K, Fox PT, Laird AR, Eickhoff SB. 2010. A link between the systems: functional differentiation and integration within the human insula revealed by meta-analysis. Brain Struct Funct 214:519-534.

Li B, Daunizeau J, Stephan KE, Penny W, Hu D, Friston K. 2011. Generalised filtering and stochastic DCM for fMRI. Neuroimage 58:442-457.

Lin QH, Liu J, Zheng YR, Liang H, Calhoun VD. 2010. Semiblind spatial ICA of fMRI using spatial constraints. Hum Brain Mapp 31:1076-1088.

Menon V. 2011. Large-scale brain networks and psychopathology: a unifying triple network model. Trends Cogn Sci 15: 483-506.

Mesulam MM, Mufson EJ. 1982a. Insula of the old world monkey. I. Architectonics in the insulo-orbito-temporal component of the paralimbic brain. J Comp Neurol 212:1-22.

Mesulam MM, Mufson EJ. 1982b. Insula of the old world monkey. III: Efferent cortical output and comments on function. J Comp Neurol 212:38-52.

Miller EK, Cohen JD. 2001. An integrative theory of prefrontal cortex function. Ann Rev Neurosci 24:167-202.

Mufson EJ, Mesulam MM. 1982. Insula of the old world monkey. II: Afferent cortical input and comments on the claustrum. J Comp Neurol 212:23-37.

Oveisgharna S, Hachinski V. 2010. Hypertension, executive dysfunction, and progression to dementia. Arch Neurol 67: 187-192.

Petrides M, Pandya DN. 1999. Dorsolateral prefrontal cortex: comparative cytoarchitectonic analysis in the human and the macaque brain and corticocortical connection patterns. Eur J Neurosci 11:1011-1036. 
Power JD, Cohen AL, Nelson SM, Wig GS, Barnes KA, Church JA, et al. 2011. Functional network organization of the human brain. Neuron 72:665-678.

Raichle ME. 2015. The restless brain: how intrinsic activity organizes brain function. Philos Trans R Soc Lond B Biol Sci 370:20140172.

Seeley WW, Menon V, Schatzberg AF, Keller J, Glover GH, Kenna H, et al. 2007. Dissociable intrinsic connectivity networks for salience processing and executive control. J Neurosci 27:2349-2356.

Shirer WR, Ryali S, Rykhlevskaia E, Menon V, Greicius MD. 2012. Decoding subject-driven cognitive states with wholebrain connectivity patterns. Cereb Cortex 22:158-165.

Smith SM, Miller KL, Salimi-Khorshidi G, Webster M, Beckmann CF, Nichols TE, et al. 2011. Network modelling methods for fMRI. Neuroimage 54:875-891.

Sridharan D, Levitin DJ, Menon V. 2008. A critical role for the right fronto-insular cortex in switching between centralexecutive and default-mode networks. Proc Natl Acad Sci U S A 105:12569-12574.

Stephan KE, Kasper L, Harrison LM, Daunizeau J, den Ouden HE, Breakspear M, Friston KJ. 2008. Nonlinear dynamic causal models for fMRI. Neuroimage 42:649-662.

Stephan KE, Penny WD, Daunizeau J, Moran RJ, Friston KJ. 2009. Bayesian model selection for group studies. Neuroimage 46:1004-1017.

Stephan KE, Penny WD, Moran RJ, den Ouden HE, Daunizeau J, Friston KJ. 2010. Ten simple rules for dynamic causal modeling. Neuroimage 49:3099-3109.

Touroutoglou A, Bliss-Moreau E, Zhang J, Mantini D, Vanduffel W, Dickerson BC, Barrett LF. 2016. A ventral salience network in the macaque brain. Neuroimage 132:190-197.

Touroutoglou A, Hollenbeck M, Dickerson BC, Feldman Barrett L. 2012. Dissociable large-scale networks anchored in the right anterior insula subserve affective experience and attention. Neuroimage 60:1947-1958.
Tsvetanov KA, Henson RN, Tyler LK, Razi A, Geerligs L, Ham TE, Rowe JB, Cambridge Centre for Ageing and Neuroscience. 2016. Extrinsic and intrinsic brain network connectivity maintains cognition across the lifespan despite accelerated decay of regional brain activation. $\mathrm{J}$ Neurosci 36:3115-3126.

Uddin LQ. 2015. Salience processing and insular cortical function and dysfunction. Nat Rev Neurosci 16:55-61.

Uddin LQ, Menon V. 2009. The anterior insula in autism: underconnected and under-examined. Neurosci Biobehav Rev 33: 1198-1203.

Uddin LQ, Supekar K, Amin H, Rykhlevskaia E, Nguyen DA, Greicius MD, Menon V. 2010. Dissociable connectivity within human angular gyrus and intraparietal sulcus: evidence from functional and structural connectivity. Cereb Cortex 20:2636-2646.

Ullsperger M, Harsay HA, Wessel JR, Ridderinkhof KR. 2010. Conscious perception of errors and its relation to the anterior insula. Brain Struct Funct 214:629-643.

Vincent JL, Kahn I, Snyder AZ, Raichle ME, Buckner RL. 2008. Evidence for a frontoparietal control system revealed by intrinsic functional connectivity. J Neurophysiol 100: 3328-3342.

Watson KK, Jones TK, Allman JM. 2006. Dendritic architecture of the von Economo neurons. Neuroscience 141:1107-1112.

Address correspondence to: Ganesh B. Chand

Division of Geriatrics and General Internal Medicine Department of Medicine Emory University School of Medicine 1841 Clifton Road NE, Suite 544 Atlanta, GA 30329

E-mail: ganesh.chand@emory.edu 